\title{
Application of the Renormalization-group Method to the Reduction of Transport Equations
}

\author{
Teiji Kunihiro $^{1}$ and Kyosuke Tsumura ${ }^{2}$ \\ ${ }^{1}$ Yukawa Institute for Theoretical Physics, Kyoto University, Sakyo-ku, Kyoto 606-8502, Japan \\ ${ }^{2}$ Department of Physics, Kyoto University, Sakyo-ku, Kyoto 606-8502, Japan
}

\begin{abstract}
We first give a comprehensive review of the renormalization group method for global and asymptotic analysis, putting an emphasis on the relevance to the classical theory of envelopes and on the importance of the existence of invariant manifolds of the dynamics under consideration. We clarify that an essential point of the method is to convert the problem from solving differential equations to obtaining suitable initial (or boundary) conditions: The RG equation determines the slow motion of the would-be integral constants in the unperturbative solution on the invariant manifold. The RG method is applied to derive the Navier-Stokes equation from the Boltzmann equation, as an example of the reduction of dynamics. We work out to obtain the transport coefficients in terms of the one-body distribution function.
\end{abstract}

\section{Introduction}

The concept of the RG was introduced by Stuckelberg and Petermann as well as Gell-Mann and Low [1] in relation to an ambiguity in the renomalization procedure of the perturbation series in quantum field theory (QFT). However, the essential nature of the RG is exact and hence nonperturbative, which was revealed and emphasized by Wilson [2]. Subsequently, as is well known, the machinery of the RG has been applied to various problems in QFT and statistical physics with a great success. [1]

The essence of the RG in quantum field theory (QFT) and statistical physics may be stated as follows: Let $\Gamma(\phi, \boldsymbol{g}(\Lambda), \Lambda)$ be the effective action (or thermodynamical potential) obtained by integration of the field variable with the energy scale down to $\Lambda$ from infinity or a very large cutoff $\Lambda_{0}$. Here $\boldsymbol{g}(\Lambda)$ is a collection of the coupling constants including the wave-function renormalization constant defined at the energy scale at $\Lambda$. Then the RG equation may be expressed as a simple fact that the effective action as a functional of the field variable $\phi$ should be the same, irrespective to how much the integration of the field variable is achieved, i.e.,

$$
\Gamma(\phi, \boldsymbol{g}(\Lambda), \Lambda)=\Gamma\left(\phi, \boldsymbol{g}\left(\Lambda^{\prime}\right), \Lambda^{\prime}\right) .
$$

If we take the limit $\Lambda^{\prime} \rightarrow \Lambda$, we have

$$
\frac{d \Gamma(\phi, \boldsymbol{g}(\Lambda), \Lambda)}{d \Lambda}=0
$$

which is the Wilson RG equation [2], or the flow equation in the Wegner's terminology [3]; notice that Eq.(1.2) is rewritten as

$$
\frac{\partial \Gamma}{\partial \boldsymbol{g}} \cdot \frac{d \boldsymbol{g}}{d \Lambda}=-\frac{\partial \Gamma}{\partial \Lambda} .
$$

If the number of the coupling constants is finite, the theory is called renormalizable. In this case, the functional space of the theory does not change in the flow given by the variation of $\Lambda$. 
Owing to the very non-perturbative nature, the RG has at least two merits: (A) Resummation of the perturbation series Applying the RG equation of Gell-Mann-Low type [1] to perturbative calculations up to first lowest orders, a resummation in the infinite order of diagrams of some kind can be achieved. That is, the RG method gives a powerful resummation method 4 . (B) Construction of infrared effective actions The RG of Wilson type 2] provides us with a systematic method for constructing low-energy effective actions which are asymptotically valid in the low-frequency and long-wave length limit.

An appearance of diverging series is a common phenomenon in every mathematical sciences not restricted in QFT, and some convenient "resummation" methods are need and developed [5]. Deducing a slow and long-wave length motion is one of the basic problems in almost all the fields of physics; for example, statistical physics including the physics of the pattern formation and the theories of collective motion in a many body system. The problems may be collectively called the reduction problem of dynamics. The RG method 6, 7, 8, 9, 10, 11, 12, might be a unified method for the reduction of dynamics as well as a powerful resummation method.

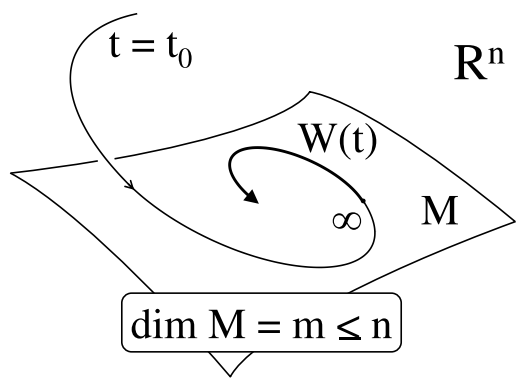

Figure 1: The geometrical image of the reduction of the dynamics. The dynamical variable $\mathbf{W}(t)$ in the $n$-dimensional phase space approaches to and after some time is eventually confined in the well-defined manifold $\mathrm{M}$ as $t$ increases.

It is noteworthy that one can draw a clear geometrical image for the reduction of dynamical systems. Let $\mathbf{W}(t)$ be an $n$-dimensional dynamical system governed by the evolution equation,

$$
\frac{d \mathbf{W}}{d t}=\mathbf{F}(\mathbf{W}, t)
$$

where $\mathbf{F}$ is an $n$-dimensional vector; the dimension $n$ may be finite or infinite. When the dynamics is reduced to an $m$-dimensional system with $m$ being smaller than or equal to $n$, the vector $\mathbf{W}(t)$ approaches a well-defined $m$-dimensional manifold $\mathrm{M}$ embedded in the $n$-dimensional phase space, as shown in Fig.1; then the geometrical object $M$ is called an attractive manifold. If after some time the $\mathbf{W}(t)$ is confined in the manifold $\mathrm{M}, \mathrm{M}$ is called an invariant manifold. Furthermore, when the dynamics on $\mathrm{M}$ is slow, $\mathrm{M}$ is also called a slow manifold. Let any point $\mathbf{W}$ within the manifold $\mathrm{M}$ be given by the relation $\mathbf{W}=\boldsymbol{R}(\boldsymbol{s})$ with an $m$-dimensional parameter $\boldsymbol{s}(t)$. Then the reduced dynamics is given by

$$
\frac{d s}{d t}=\boldsymbol{G}(s), \quad \mathbf{W}=\boldsymbol{R}(s)
$$

where the first equation with the vector field $\boldsymbol{G}(\boldsymbol{s})$ defined on $\mathrm{M}$ gives the reduced dynamics within the manifold $\mathrm{M}$ and the second is the representation of $\mathrm{M}$. In the quantum field theory, the dynamical variable $\mathbf{W}$ corresponds to the set of coupling constants and the renomalizability may be interpreted as the existence of an invariant manifold in the space of coupling constants; 
the increasing time $t$ and the vector field $\boldsymbol{G}(\boldsymbol{s})$ correspond to the decreasing energy cutoff $\Lambda$ and the $\beta$ function, respectively.

In this article, (1) we show that the RG gives a powerful and systematic method for the reduction of dynamics and also provides a transparent way for the construction of the attractive slow manifold. (2) we apply the method to have the fluid dynamical limit of the Boltzmann equation as an example of the reduction of dynamics and the construction of the slow manifold 12 . We derive the Navier-Stokes equation explicitly from the Boltzmann equation for the first time; the microscopic expressions of the transport coefficients are given. (3) We will put an emphasis on the relation of the underlying mathematics of the $R G$ method with the classical theory of envelopes in mathematical analysis [7, 8, 9, 10, 13.

\section{The RG method and the classical theory of envelopes}

We here give a brief review of the theory of envelopes. Although the theory can be formulated in higher dimensions [7, 8, 9, we consider here envelope curves, for simplicity.

Let $\left\{C_{\tau}\right\}_{\tau}$ be a family of curves parameterized by $\tau$ in the $x-y$ plane; here $C_{\tau}$ is represented by the equation $F(x, y, \tau)=0$. We suppose that $\left\{C_{\tau}\right\}_{\tau}$ has the envelope $E$, which is represented by the equation $G(x, y)=0$, as shown in Fig.2. The problem is to obtain $G(x, y)$ from $F(x, y, \tau)$.

Now let $E$ and a curve $C_{\tau_{0}}$ have the common tangent line at $(x, y)=\left(x_{0}, y_{0}\right)$, i.e., $\left(x_{0}, y_{0}\right)$ is the point of tangency. Then $x_{0}$ and $y_{0}$ are functions of $\tau_{0} ; x_{0}=\phi\left(\tau_{0}\right), y_{0}=\psi\left(\tau_{0}\right)$, and of course $G\left(x_{0}, y_{0}\right)=0$. Conversely, for each point $\left(x_{0}, y_{0}\right)$ on $E$, there exists a parameter $\tau_{0}$. So we can reduce the problem to get $\tau_{0}$ as a function of $\left(x_{0}, y_{0}\right)$; then $G(x, y)$ is obtained as $F(x, y, \tau(x, y))=G(x, y)$. Notice that since there is a relation $G\left(x_{0}, y_{0}\right)=0$ between $x_{0}$ and $y_{0}$, $\tau_{0}$ is actually a function of $x_{0}$ or $y_{0} . \tau_{0}\left(x_{0}, y_{0}\right)$ can be obtained as follows.

Since the tangent line of $E$ at $\left(x_{0}, y_{0}\right)$ is perpendicular to the normal direction of $F(x, y, \tau)=0$ at the same point, one has $F_{x}\left(x_{0}, y_{0}, \tau_{0}\right) \phi^{\prime}\left(\tau_{0}\right)+F_{y}\left(x_{0}, y_{0}, \tau_{0}\right) \psi^{\prime}\left(\tau_{0}\right)=0$. On the other hand, differentiating $F\left(x\left(\tau_{0}\right), y\left(\tau_{0}\right), \tau_{0}\right)=0$ with respect to $\tau_{0}$, one also has $F_{x}\left(x_{0}, y_{0}, \tau_{0}\right) \phi^{\prime}\left(\tau_{0}\right)+F_{y}\left(x_{0}, y_{0}, \tau_{0}\right) \psi^{\prime}\left(\tau_{0}\right)+$ $F_{\tau_{0}}\left(x_{0}, y_{0}, \tau_{0}\right)=0$. Combining the last two equations, we have

$$
F_{\tau_{0}}\left(x_{0}, y_{0}, \tau_{0}\right) \equiv \frac{\partial F\left(x_{0}, y_{0}, \tau_{0}\right)}{\partial \tau_{0}}=0 .
$$

This is the basic equation of the theory of envelopes. Notice that the envelope equation has the similar form as the RG equation.

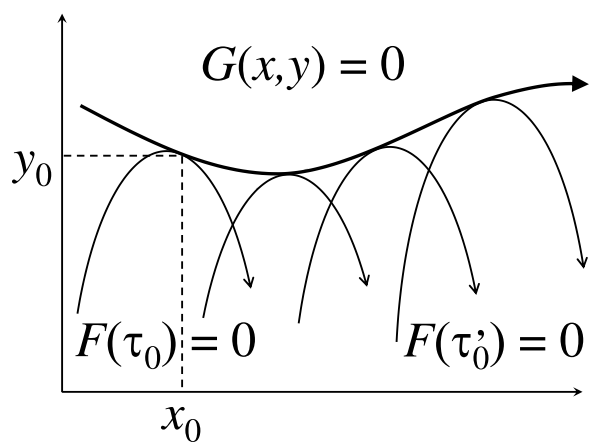

Figure 2: A family of curves $F\left(x, y, \tau_{0}\right)=0$ parameterized with $\tau_{0}$ and its envelope defined by $G(x, y)=0$. 
One can thus eliminate the parameter $\tau_{0}$ to get a relation between $x_{0}$ and $y_{0} ; G(x, y)=$ $F\left(x, y, \tau_{0}(x, y)\right)=0$, with the replacement $\left(x_{0}, y_{0}\right) \rightarrow(x, y) . G(x, y)$ is called the discriminant of $F(x, y, t)$.

When the function $F$ has an additional dependence on a vector $\boldsymbol{C}(\tau)$, i.e., $F=F(x, y, \tau, \boldsymbol{C}(\tau))$, the envelope equation reads

$$
F_{\tau_{0}}\left(x_{0}, y_{0}, \tau_{0}, \boldsymbol{C}\left(\tau_{0}\right)\right) \equiv \frac{\partial F\left(x_{0}, y_{0}, \tau_{0}\right)}{\partial \tau_{0}}+\frac{\partial \boldsymbol{C}}{\partial \tau_{0}} \frac{\partial F\left(x_{0}, y_{0}, \tau_{0}, \boldsymbol{C}\left(\tau_{0}\right)\right)}{\partial \boldsymbol{C}}=0
$$

Comments are in order here: (1) When the family of curves is given by the function $y=f(x, \tau)$, the envelope equation is reduced to $\partial f / \partial \tau_{0}=0$; the envelope is given by $y=f\left(x, \tau_{0}(x)\right)$. (2) The equation $G(x, y)=0$ may give not only the envelope $E$ but also a set of singularities of the curves $\left\{C_{\tau}\right\}_{\tau}$.

\section{The RG method; a simplest example}

In this section, using a simplest example we show how the RG method works for obtaining global and asymptotic behavior of solutions of differential equations. We shall present the method so that the reader will readily see that the notion of envelopes is intrinsically related to the method. We shall emphasize that an essential point of the method is tuning the initial condition at an arbitrary time $t_{0}$ perturbatively along with solving the perturbative equations successively. One will see that the reasoning for various steps in the procedure and the underlying picture are quite different from the original ones given in [6]. We believe, however, that the present formulation emphasizing the role of initial conditions and the relevance to envelopes of perturbative local solutions straightens the original argument, and is the most comprehensive one.

Let us take the following simplest example to show our method:

$$
\frac{d^{2} x}{d t^{2}}+\epsilon \frac{d x}{d t}+x=0
$$

where $\epsilon$ is supposed to be small. The solution to Eq. (3.1) reads $x(t)=\bar{A} \exp \left(-\frac{\epsilon}{2} t\right) \sin \left(\sqrt{1-\frac{\epsilon^{2}}{4}} t+\bar{\theta}\right)$, where $\bar{A}$ and $\bar{\theta}$ are constants.

Now, let us obtain the solution around the initial time $t=t_{0}$ in a perturbative way, expanding $x$ as $x\left(t, t_{0}\right)=x_{0}\left(t, t_{0}\right)+\epsilon x_{1}\left(t, t_{0}\right)+\epsilon^{2} x_{2}\left(t, t_{0}\right)+\ldots$, where $x_{n}\left(t, t_{0}\right)(n=0,1,2 \ldots)$ satisfy $\ddot{x}_{0}+x_{0}=0, \quad \ddot{x}_{n+1}+x_{n+1}=-\dot{x}_{n}$.

The initial condition may be specified by

$$
x\left(t_{0}, t_{0}\right)=W\left(t_{0}\right) .
$$

We suppose that the initial value $W\left(t_{0}\right)$ is always on an exact solution of Eq.(3.1) for any $t_{0}$. We also expand the initial value $W\left(t_{0}\right) ; W\left(t_{0}\right)=W_{0}\left(t_{0}\right)+\epsilon W_{1}\left(t_{0}\right)+\epsilon^{2} W_{2}\left(t_{0}\right)+\ldots$, and the terms $W_{i}\left(t_{0}\right)$ will be determined so that the perturbative solutions around different initial times $t_{0}$ have an envelope. Hence the initial value $W(t)$ thus constructed will give the (approximate but) global solution of the equation.

Let us perform the above program. The lowest solution may be given by $x_{0}\left(t, t_{0}\right)=A\left(t_{0}\right) \sin (t+$ $\left.\theta\left(t_{0}\right)\right)$, where we have made it explicit that the constants $A$ and $\theta$ may depend on the initial time $t_{0}$. The initial value $W\left(t_{0}\right)$ as a function of $t_{0}$ is specified as $W_{0}\left(t_{0}\right)=x_{0}\left(t_{0}, t_{0}\right)=A\left(t_{0}\right) \sin \left(t_{0}+\theta\left(t_{0}\right)\right)$. We remark that the zero-th order solution a neutrally stable solution; with the perturbation 
$\epsilon \neq 0$ the constants $A$ and $\theta$ may move slowly. We shall see that the envelope equation gives the equations describing the slow motion of $A$ and $\theta$.

The first order equation now reads $\ddot{x}_{1}+x_{1}=-A \cos (t+\theta)$, and we choose the solution in the following form, $x_{1}\left(t, t_{0}\right)=-\frac{A}{2} \cdot\left(t-t_{0}\right) \sin (t+\theta)$, which means that the first order initial value $W_{1}\left(t_{0}\right)=0$ so that the lowest order value $W_{0}\left(t_{0}\right)$ approximates the exact value as closely as possible. Similarly, the second order solution may be given by $x_{2}(t)=\frac{A}{8}\left\{\left(t-t_{0}\right)^{2} \sin (t+\theta)-(t-\right.$ $\left.\left.t_{0}\right) \cos (t+\theta)\right\}$, thus $W_{2}\left(t_{0}\right)=0$ again for the present linear equation.

It should be noted that the secular terms have appeared in the higher order terms, which are absent in the exact solution and invalidates the perturbation theory for $t$ far from $t_{0}$. However, with the very existence of the secular terms, we could make $W_{i}\left(t_{0}\right)(i=1,2)$ vanish and $W\left(t_{0}\right)=W_{0}\left(t_{0}\right)$ up to the third order.

Collecting the terms, we have

$$
\begin{aligned}
x\left(t, t_{0}\right)= & A \sin (t+\theta)-\epsilon \frac{A}{2}\left(t-t_{0}\right) \sin (t+\theta) \\
& +\epsilon^{2} \frac{A}{8}\left\{\left(t-t_{0}\right)^{2} \sin (t+\theta)-\left(t-t_{0}\right) \cos (t+\theta)\right\},
\end{aligned}
$$

and more importantly $W\left(t_{0}\right)=W_{0}\left(t_{0}\right)=A\left(t_{0}\right) \sin \left(t_{0}+\theta\left(t_{0}\right)\right)$, up to $O\left(\epsilon^{3}\right)$. We remark that $W\left(t_{0}\right)$ describing the solution is parameterized by possibly slowly moving variable $A\left(t_{0}\right)$ and $\phi\left(t_{0}\right) \equiv t_{0}+\theta\left(t_{0}\right)$ in a definite way.

Now we have a family of curves $\left\{C_{t_{0}}\right\}_{t_{0}}$ given by functions $\left\{x\left(t, t_{0}\right)\right\}_{t_{0}}$ parameterized with $t_{0}$. They are all on the exact curve $W(t)$ at $t=t_{0}$ up to $O\left(\epsilon^{3}\right)$, but only valid locally for $t$ near $t_{0}$. So it is conceivable that the envelope $E$ of $\left\{C_{t_{0}}\right\}_{t_{0}}$ which contacts with each local solution at $t=t_{0}$ will give a global solution. Thus the envelope function $x_{E}(t)$ coincides with $W(t)$; $x_{E}(t)=x(t, t)=W(t)$.

Our task is actually to determine $A\left(t_{0}\right)$ and $\theta\left(t_{0}\right)$ as functions of $t_{0}$ so that the family of the local solutions has an envelope. According to the classical theory of envelopes given in the previous section, the above program can be achieved by imposing that the envelope equation

$$
\frac{d x\left(t, t_{0}\right)}{d t_{0}}=0
$$

gives the solution $t_{0}=t$. From Eq.'s (3.3), we have

$$
\frac{d A}{d t_{0}}+\epsilon A=0, \quad \frac{d \theta}{d t_{0}}+\frac{\epsilon^{2}}{8}=0,
$$

where we have used the fact that $d A / d t$ is $O(\epsilon)$ and neglected the terms of $O\left(\epsilon^{3}\right)$. Solving the equations, we have $A\left(t_{0}\right)=\bar{A} \mathrm{e}^{-\epsilon t_{0} / 2}$ and $\theta\left(t_{0}\right)=-\frac{\epsilon^{2}}{8} t_{0}+\bar{\theta}$, where $\bar{A}$ and $\bar{\theta}$ are constant numbers. Thus we get

$$
x_{E}(t)=x(t, t)=W_{0}(t)=\bar{A} \exp \left(-\frac{\epsilon}{2} t\right) \sin \left(\left(1-\frac{\epsilon^{2}}{8}\right) t+\bar{\theta}\right)
$$

up to $O\left(\epsilon^{3}\right)$. Noting that $\sqrt{1-\epsilon^{2} / 4}=1-\epsilon^{2} / 8+O\left(\epsilon^{4}\right)$, one finds that the resultant envelope function $x_{E}(t)=W_{0}(t)$ is an approximate but global solution to Eq.(3.1). 


\section{The RG-reduction of dynamics of a generic evolution equation}

The theory of the reduction of evolution equations must give a definite method to find out the vector field $\boldsymbol{G}(\boldsymbol{s})$ as well as to construct the attractive manifold M or the function $\boldsymbol{R}(\boldsymbol{s})$ in Eq.(1.5). It is often that these tasks can be achieved in a perturbative way as follows,

$$
\frac{d s}{d t}=\boldsymbol{G}_{0}(\boldsymbol{s})+\gamma(\boldsymbol{s}), \quad \boldsymbol{R}(\boldsymbol{s})=\boldsymbol{R}_{0}(\boldsymbol{s})+\boldsymbol{\rho}(\boldsymbol{s}),
$$

where $\boldsymbol{R}_{0}(\boldsymbol{s})$ gives the coordinate of the unperturbed invariant manifold $\mathrm{M}_{0}$ and $\boldsymbol{\rho}$ gives the deformation of the manifold by the perturbation, as shown in Fig.3. The unperturbed vector field $\boldsymbol{G}_{0}(\boldsymbol{s})$ governs the reduced dynamics on $\mathrm{M}_{0}$ and $\gamma(\boldsymbol{s})$ the modification of the dynamics. The important point lies in the fact that the modification of the manifold and the dynamics are both still function of $s$ parameterizing the unperturbed manifold $\mathrm{M}_{0}$.

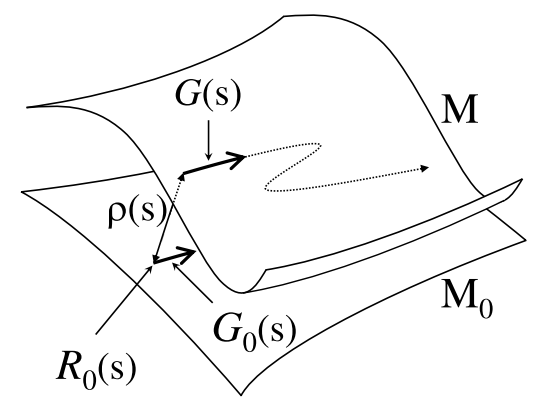

Figure 3: The geometrical image of the perturbative construction of the attractive manifold M and the reduced dynamics of the vector field $\boldsymbol{G}$ on it. $\mathrm{M}_{0}$ and $\boldsymbol{G}_{0}$ denote the unperturbed ones.

Such a view on the reduction of dynamics was emphasized by Kuramoto 14. It is remarkable that Bogoliubov gave the notion of invariant manifold in his contribution to the theory of non-linear oscillators 15; he also described the fluid dynamical limit of Boltzmann equation as a construction of a (slow) invariant manifold spanned by the hydrodynamical quantities inbeded in the functional space composed of the sigle-particle distribution function [16].

In this section, we show how the renormalization-group method works to make the reduction of the dynamics of a generic system possessing the possible reduction of dynamics in the perturbative way 11]: It will be clarified that the system reduction is accomplished by explicitly constructing the invariant manifold and the slow dynamics on the manifold in the perturbative way. We emphasize that the initial values are chosen by using a simple formula for the special solutions to differential equations as in the previous section.

We treat the following rather generic vector equations in this section:

$$
\partial_{t} \mathbf{u}=A \mathbf{u}+\epsilon \mathbf{F}(\mathbf{u})
$$

where $\partial_{t} \mathbf{u}=\partial \mathbf{u} / \partial t, A$ is a linear operator, $\mathbf{F}$ a nonlinear function of $\mathbf{u}$ and $\epsilon$ is a small parameter $(|\epsilon|<1)$. We assume that $A$ has multiply degenerated zero eigenvalues and other eigenvalues of $A$ have a negative real part. We assume that $A$ has semi-simple 0 eigenvalues in the present paper; the case when $A$ has a multidimensional Jordan cell is also treated in [1].

We are interested in constructing the attractive manifold $\mathrm{M}$ at $t \rightarrow \infty$ and the reduced dynamics on it. We try to construct solve the problem in the perturbation theory by expanding $\mathbf{u}$ as

$$
\mathbf{u}\left(t ; t_{0}\right)=\mathbf{u}_{0}\left(t ; t_{0}\right)+\epsilon \mathbf{u}_{1}\left(t ; t_{0}\right)+\epsilon^{2} \mathbf{u}_{2}\left(t ; t_{0}\right)+\cdots,
$$


with the initial value $\mathbf{W}\left(t_{0}\right)$ at an arbitrary time $t_{0}$. The equations in the first few orders read

$$
\left(\partial_{t}-A\right) \mathbf{u}_{0}=0, \quad\left(\partial_{t}-A\right) \mathbf{u}_{1}=\mathbf{F}\left(\mathbf{u}_{0}\right), \quad\left(\partial_{t}-A\right) \mathbf{u}_{2}=\mathbf{F}^{\prime}\left(\mathbf{u}_{0}\right) \mathbf{u}_{1},
$$

where

$$
\left(\mathbf{F}^{\prime}\left(\mathbf{u}_{0}\right) \mathbf{u}_{1}\right)_{i}=\sum_{j=1}^{n}\left\{\partial\left(F^{\prime}\left(\mathbf{u}_{0}\right)\right)_{i} / \partial\left(u_{0}\right)_{j}\right\}\left(u_{1}\right)_{j},
$$

is a Freche derivative for the $n$-dimensional vector.

We suppose that the equation has been solved up to $t=t_{0}$ and the solution has the value $\mathbf{W}\left(t_{0}\right)$ at $t_{0}$. Actually, the initial value must be determined by the perturbative solution self-consistently; indeed, $\mathbf{u}(t)=\mathbf{W}(t)$ is the solution to (4.2) in the global domain. Therefore it should be also expanded as follows; $\mathbf{W}\left(t_{0}\right)=\mathbf{W}_{0}\left(t_{0}\right)+\epsilon \mathbf{W}_{1}\left(t_{0}\right)+\epsilon^{2} \mathbf{W}_{2}\left(t_{0}\right)+\cdots=\mathbf{W}_{0}\left(t_{0}\right)+\boldsymbol{\rho}\left(t_{0}\right)$, where $\boldsymbol{\rho}\left(t_{0}\right)$ is supposed to be an independent function of $\mathbf{W}_{0}$. They are not yet known at present but will be determined so that the perturbative expansion becomes valid. One of the main purposes in this section is to show how sensibly the initial values can be determined order by order.

In the present paper, we confine ourselves to the case where $A$ has semi-simple 0 eigenvalues. Let the dimension of $\operatorname{ker} A$ be $m ; A \mathbf{U}_{i}=0,(i=1,2, \ldots, m)$. We suppose that other eigenvalues have negative real parts; $A \mathbf{U}_{\alpha}=\lambda_{\alpha} \mathbf{U}_{\alpha}, \quad(\alpha=m+1, m+2, \cdots, n)$, where $\operatorname{Re} \lambda_{\alpha}<0$. One may assume without loss of generality that $\mathbf{U}_{i}$ 's and $\mathbf{U}_{\alpha}$ 's are linearly independent.

The adjoint operator $A^{\dagger}$ has the same eigenvalues as $A$ has; $A^{\dagger} \tilde{\mathbf{U}}_{i}=0, \quad(i=1,2, \ldots, m)$ and $A^{\dagger} \tilde{\mathbf{U}}_{\alpha}=\lambda_{\alpha}^{*} \tilde{\mathbf{U}}_{\alpha},(\alpha=m+1, m+2, \cdots, n)$. Here we suppose that $\tilde{\mathbf{U}}_{i}$ ' and $\tilde{\mathbf{U}}_{\alpha}$ 's are linearly independent. Without loss of generality, one can choose the eigenvectors so that $\left\langle\tilde{\mathbf{U}}_{i}, \mathbf{U}_{\alpha}\right\rangle=0=$ $\left\langle\tilde{\mathbf{U}}_{\alpha}, \mathbf{U}_{i}\right\rangle$, with $1 \leq i \leq m$ and $m+1 \leq \alpha \leq n$. We denote the projection operators by $P$ and $Q$ which projects onto the kernel of $A$ and the space orthogonal to $\operatorname{Ker} A$, respectively.

Since we are interested in the asymptotic state as $t \rightarrow \infty$, we may assume that the lowest-order initial value belongs to $\operatorname{Ker} A$ :

$$
\mathbf{W}_{0}\left(t_{0}\right)=\sum_{i=1}^{m} C_{i}\left(t_{0}\right) \mathbf{U}_{i}=\mathbf{W}_{0}[\boldsymbol{C}] .
$$

Thus trivially, $\mathbf{u}_{0}\left(t ; t_{0}\right)=\mathrm{e}^{\left(t-t_{0}\right) A} \mathbf{W}_{0}\left(t_{0}\right)=\sum_{i=1}^{m} C_{i}\left(t_{0}\right) \mathbf{U}_{i}$. We notice that a natural parameterization of the invariant manifold in the lowest order $\mathrm{M}_{0}$ is given by the set of the integral constants $\boldsymbol{C}={ }^{t}\left(C_{1}, C_{2}, \cdots, C_{m}\right)$ being varied.

The first order equation (4.4) with the initial value $\mathbf{W}_{1}\left(t_{0}\right)$ which is not yet determined is formally solved to be

$$
\begin{aligned}
\mathbf{u}_{1}\left(t ; t_{0}\right)= & \mathrm{e}^{\left(t-t_{0}\right) A}\left[\mathbf{W}_{1}\left(t_{0}\right)+A^{-1} Q \mathbf{F}\left(\mathbf{W}_{0}\left(t_{0}\right)\right)\right] \\
& +\left(t-t_{0}\right) P \mathbf{F}\left(\mathbf{W}_{0}\left(t_{0}\right)\right)-A^{-1} Q \mathbf{F}\left(\mathbf{W}_{0}\left(t_{0}\right)\right) .
\end{aligned}
$$

The first term has a possibility to give rise to a fast motion, which should be avoided and are analogous to divergent terms in quantum field theory; the divergent terms are subtracted away by counter terms, which are analogue to the initial values $\mathbf{W}_{i}$ here. Indeed it is nice that the initial value $\mathbf{W}_{1}\left(t_{0}\right)$ not yet determined can be chosen so as to cancel out the would-be fast term as follows; $\mathbf{W}_{1}\left(t_{0}\right)=-A^{-1} Q \mathbf{F}\left(\mathbf{W}_{0}\left(t_{0}\right)\right)$, which satisfies $P \mathbf{W}_{1}\left(t_{0}\right)=0$ and is a function solely of $\boldsymbol{C}\left(t_{0}\right)$. Thus we have for the first order solution, $\mathbf{u}_{1}\left(t ; t_{0}\right)=\left(t-t_{0}\right) P \mathbf{F}-A^{-1} Q \mathbf{F}$, where the argument of $\mathbf{F}$ is $\mathbf{W}_{0}[\boldsymbol{C}]$. Now the invariant manifold is modified to $\mathrm{M}_{1}$ given by

$$
\mathrm{M}_{1}=\left\{\mathbf{u} \mid \mathbf{u}=\mathbf{W}_{0}-\epsilon A^{-1} Q \mathbf{F}\left(\mathbf{W}_{0}\right)\right\}
$$


If one stops to this order, the approximate solution reads

$$
\mathbf{u}\left(t ; t_{0}\right)=\mathbf{W}_{0}+\epsilon\left\{\left(t-t_{0}\right) P \mathbf{F}-A^{-1} Q \mathbf{F}\right\} .
$$

Then the RG equation $\partial \mathbf{u} /\left.\partial t_{0}\right|_{t_{0}=t}=0$ gives $\dot{\mathbf{W}}_{0}(t)=\epsilon P \mathbf{F}\left(\mathbf{W}_{0}(t)\right)$, which is reduced to an $m$-dimensional coupled equation,

$$
\dot{C}_{i}(t)=\epsilon\left\langle\tilde{\mathbf{U}}_{i}, \mathbf{F}\left(\mathbf{W}_{0}[\boldsymbol{C}]\right)\right\rangle, \quad(i=1,2, \cdots, m) .
$$

One now sees that $\epsilon P \mathbf{F}\left(\mathbf{W}_{0}[\boldsymbol{C}]\right)$ gives the vector filed $\boldsymbol{G}_{0}(\boldsymbol{s})$ with $\boldsymbol{C}$ being identified with $\boldsymbol{s}$ in Eq.(4.1). The global solution representing a trajectory on the invariant manifold up to this order is given by

$$
\mathbf{u}(t)=\mathbf{u}\left(t ; t_{0}=t\right)=\sum_{i=1}^{m} C_{i}(t) \mathbf{U}_{i}-\epsilon A^{-1} Q \mathbf{F}\left(\mathbf{W}_{0}[\boldsymbol{C}]\right)
$$

with $\boldsymbol{C}(t)$ being the solution to (4.10).

In short, we have derived the invariant manifold as the initial value represented by (4.11) and the reduced dynamics (4.10) on it in the $R G$ method in the first order approximation.

The above procedure can be easily extended to second and higher orders and the modification of the vector field $\gamma(s)$ in Eq.(4.1) is readily obtained, as shown in [11].

\section{Fluid dynamical limit of Boltzmann equation}

In this section, we apply the RG method formulated in the previous sections and in [11] to obtain the fluid dynamical limit of the Boltzmann equation 21. This is an example of reducing a kinetic equation to a slower dynamics 12. In this article, we complete the derivation of the Navier-Stokes equation with some corrections to the previous treatment 12; we shall also work out to give the explicit formulae of the transport coefficients.

\subsection{Basics of the Boltzmann Equation}

The Boltzmann equation [21, 20] is a transport equation which describes the time evolution of one-particle distribution function defined in the phase space:

$$
\frac{\partial}{\partial t} f(\boldsymbol{r}, \boldsymbol{v}, t)+\boldsymbol{v} \cdot \boldsymbol{\nabla} f(\boldsymbol{r}, \boldsymbol{v}, t)=I[f](\boldsymbol{r}, \boldsymbol{v}, t) .
$$

The right-hand side of the above equation is called the collision integral,

$$
I[f](\boldsymbol{r}, \boldsymbol{v}, t)=\int \boldsymbol{d}^{\mathbf{3}} \boldsymbol{v}_{\mathbf{1}} \int \boldsymbol{d}^{\mathbf{3}} \boldsymbol{v}_{\mathbf{2}} \int \boldsymbol{d}^{\mathbf{3}} \boldsymbol{v}_{\mathbf{3}} \omega\left(\boldsymbol{v}, \boldsymbol{v}_{\mathbf{1}} \mid \boldsymbol{v}_{\mathbf{2}}, \boldsymbol{v}_{\mathbf{3}}\right)\left(f\left(\boldsymbol{r}, \boldsymbol{v}_{\mathbf{2}}, t\right) f\left(\boldsymbol{r}, \boldsymbol{v}_{\mathbf{3}}, t\right)-f(\boldsymbol{r}, \boldsymbol{v}, t) f\left(\boldsymbol{r}, \boldsymbol{v}_{\mathbf{1}}, t\right)\right),(5 .
$$

where $\omega\left(\boldsymbol{v}, \boldsymbol{v}_{\mathbf{1}} \mid \boldsymbol{v}_{\mathbf{2}}, \boldsymbol{v}_{\mathbf{3}}\right)$ denotes the transition probability which comes from a microscopic twoparticle interaction. We remark that the transition probability $\omega\left(\boldsymbol{v}, \boldsymbol{v}_{\mathbf{1}} \mid \boldsymbol{v}_{\mathbf{2}}, \boldsymbol{v}_{\mathbf{3}}\right)$ contains delta functions reflecting energy-momentum conservation law, and satisfies the following relations based on the indistinguishability of identical particles and the time reversal symmetry in the scattering process: $\omega\left(\boldsymbol{v}, \boldsymbol{v}_{\mathbf{1}} \mid \boldsymbol{v}_{\mathbf{2}}, \boldsymbol{v}_{\mathbf{3}}\right)=\omega\left(\boldsymbol{v}_{\mathbf{1}}, \boldsymbol{v} \mid \boldsymbol{v}_{\mathbf{3}}, \boldsymbol{v}_{\mathbf{2}}\right)=\omega\left(\boldsymbol{v}_{\mathbf{2}}, \boldsymbol{v}_{\mathbf{3}} \mid \boldsymbol{v}, \boldsymbol{v}_{\mathbf{1}}\right)=\omega\left(\boldsymbol{v}_{\mathbf{3}}, \boldsymbol{v}_{\mathbf{2}} \mid \boldsymbol{v}_{\mathbf{1}}, \boldsymbol{v}\right)$.

To make explicit the correspondence to the general formulation given in the previous section 11 , one may treat the argument $\boldsymbol{v}$ as a discrete variable 14. Discriminating the arguments $(\boldsymbol{r}, t)$ and 
$\boldsymbol{v}$, we use $\boldsymbol{v}$ as a subscript for the distribution function: $f(\boldsymbol{r}, \boldsymbol{v}, t)=f \boldsymbol{v}(\boldsymbol{r}, t) \equiv[\boldsymbol{f}(\boldsymbol{r}, t)]_{\boldsymbol{v}}$. Then the Boltzmann equation now reads

$$
\frac{\partial}{\partial t} f_{\boldsymbol{v}}(\boldsymbol{r}, t)+\boldsymbol{v} \cdot \boldsymbol{\nabla} f_{\boldsymbol{v}}(\boldsymbol{r}, t)=I[f] \boldsymbol{v}(\boldsymbol{r}, t)
$$

where

$$
I[f] \boldsymbol{v}(\boldsymbol{r}, t) \equiv \sum_{\boldsymbol{v}_{\mathbf{1}}} \sum_{\boldsymbol{v}_{\mathbf{2}}} \sum_{\boldsymbol{v}_{\mathbf{3}}} \omega\left(\boldsymbol{v}, \boldsymbol{v}_{\mathbf{1}} \mid \boldsymbol{v}_{\mathbf{2}}, \boldsymbol{v}_{\mathbf{3}}\right)\left(f \boldsymbol{v}_{\mathbf{2}}(\boldsymbol{r}, t) f \boldsymbol{v}_{\mathbf{3}}(\boldsymbol{r}, t)-f \boldsymbol{v}(\boldsymbol{r}, t) f \boldsymbol{v}_{\mathbf{1}}(\boldsymbol{r}, t)\right)
$$

As promised, we apply the RG method to extract the low-frequency dynamics from a given kinetic equation; in other words, we achieve the coarse-graining of temporal scale by the RG method. We introduce $\epsilon$ in front of spatial derivative of the Boltzmann equation to make the application of the perturbation theory possible:

$$
\frac{\partial}{\partial t} f \boldsymbol{v}(\boldsymbol{r}, t)=I[f] \boldsymbol{v}(\boldsymbol{r}, t)-\epsilon \boldsymbol{v} \cdot \boldsymbol{\nabla} f \boldsymbol{v}(\boldsymbol{r}, t)
$$

\subsection{Procedure 1 : invariant manifold \& approximate solution}

As was done in the previous sections, we first expand the initial values as follows:

$$
f_{\boldsymbol{v}}\left(\boldsymbol{r}, t_{0}\right)=f_{\boldsymbol{v}}^{(0)}\left(\boldsymbol{r}, t_{0}\right)+\epsilon f_{\boldsymbol{v}}^{(1)}\left(\boldsymbol{r}, t_{0}\right)+\epsilon^{2} f_{\boldsymbol{v}}^{(2)}\left(\boldsymbol{r}, t_{0}\right)+\cdots
$$

Then let $\tilde{f} \boldsymbol{v}\left(\boldsymbol{r}, t ; t_{0}\right)$ be an approximate solution around $t=t_{0}$, which obeys Eq. (5.5) with the initial condition at $t=t_{0}: \tilde{f}_{\boldsymbol{v}}\left(\boldsymbol{r}, t_{0} ; t_{0}\right)=f_{\boldsymbol{v}}\left(\boldsymbol{r}, t_{0}\right)$. We try to solve $\tilde{f} \boldsymbol{v}\left(\boldsymbol{r}, t ; t_{0}\right)$ by the perturbation theory by expanding it as

$$
\tilde{f} \boldsymbol{v}\left(\boldsymbol{r}, t ; t_{0}\right)=\tilde{f}_{\boldsymbol{v}}^{(0)}\left(\boldsymbol{r}, t ; t_{0}\right)+\epsilon \tilde{f}_{\boldsymbol{v}}^{(1)}\left(\boldsymbol{r}, t ; t_{0}\right)+\epsilon^{2} \tilde{f}_{\boldsymbol{v}}^{(2)}\left(\boldsymbol{r}, t ; t_{0}\right)+\cdots,
$$

with the respective initial conditions; $\tilde{f}_{\boldsymbol{v}}^{(\mu)}\left(\boldsymbol{r}, t_{0} ; t_{0}\right)=f_{\boldsymbol{v}}^{(\mu)}\left(\boldsymbol{r}, t_{0}\right)$ for $\mu=0,1,2, \cdots$. Substituting the above expansion in (5.5), we obtain the series of the perturbative equations. The lowest few equations read

$$
\begin{aligned}
\frac{\partial}{\partial t} \tilde{f}_{\boldsymbol{v}}^{(0)} & =\left.I[f] \boldsymbol{v}\right|_{\boldsymbol{f}=\tilde{\boldsymbol{f}}^{(0)}}, \\
\frac{\partial}{\partial t} \tilde{f}_{\boldsymbol{v}}^{(1)} & =\left.\sum_{\boldsymbol{k}} \frac{\partial}{\partial f_{\boldsymbol{k}}} I[f] \boldsymbol{v}\right|_{\boldsymbol{f}=\tilde{\boldsymbol{f}}^{(0)}} \tilde{f}_{\boldsymbol{k}}^{(1)}-\boldsymbol{v} \cdot \nabla \tilde{f}_{\boldsymbol{v}}^{(0)} \\
\frac{\partial}{\partial t} \tilde{f}_{\boldsymbol{v}}^{(2)} & =\left.\sum_{\boldsymbol{k}} \frac{\partial}{\partial f_{\boldsymbol{k}}} I[f] \boldsymbol{v}\right|_{\boldsymbol{f}=\tilde{\boldsymbol{f}}^{(0)}} \tilde{f}_{\boldsymbol{k}}^{(2)}-\boldsymbol{v} \cdot \nabla \tilde{f}_{\boldsymbol{v}}^{(1)}
\end{aligned}
$$

Here a remark is in order: We have actually used the linearized Boltzmann equation [20] neglecting the second-order term of $\tilde{\boldsymbol{f}}^{(1)}$ in (5.10). It is known that the neglected term produces the so-called Burnett terms which are absent in the usual Navier-Stokes equations [22].

\subsection{Procedure 2 : order-by-order analysis}

Here we summarize the significance of each order of equation: 
- 0th order

The 0th-order equation is in general a nonlinear algebraic equation which determines the lowest-order invariant manifold $M_{0}\left(\equiv \tilde{\boldsymbol{f}}^{(0)}\right)$. The special solution of this equation includes some integration constants. It turns out that these would-be constants become the slow variables on $M_{0}$ by the RG equation.

- 1st order

The 1st-order equation is a linear differential equation because we consider the dynamics near $M_{0}$. First we derive the zero modes from the eigen vectors of its time evolution operator. Then we define the appropriate inner product and projection operator to the kernel space spanned by the zero modes. Using these definitions, we solve the linear differential equation. The condition that the fast modes orthogonal to the zero modes vanish determine the initial condition in this order and thereby the 1st-order invariant manifold $M_{1}\left(\equiv \tilde{\boldsymbol{f}}^{(1)}\right)$; the 1storder perturbation gives the deformation of the invariant manifold from $M_{0}$ to $M_{1}$. The coordinates to describe the slow modes are still defined on $M_{0}$.

- 2nd and higher orders

The 2nd-order equation is a linear differential equation with the same time evolution operator. We can solve the 2nd-order equation with the same procedure as the 1 st one. The 2nd-order invariant manifold $M_{2}\left(\equiv \tilde{\boldsymbol{f}}^{(2)}\right)$ is determined in the same manner as in the 1st order. This procedure is able to be continued up to arbitrary orders. Although the successive deformation of the invariant manifold is obtained, the coordinates for the slow variables are still on $M_{0}$.

Now we are interested in the slow motion which may be realized asymptotically as $t \rightarrow \infty$. Therefore we put the stationary condition on (5.8) and the 0th-order equation becomes the following nonlinear algebraic equation:

$$
\frac{\partial}{\partial t} \tilde{f}_{\boldsymbol{v}}^{(0)}=\left.0 \Longrightarrow I[f] \boldsymbol{v}\right|_{\boldsymbol{f}=\tilde{\boldsymbol{f}}^{(0)}}=0
$$

The 0th-order approximate solution $\tilde{\boldsymbol{f}}^{(0)}$ is the fixed point of the collision integral, and thus is a local equilibrium distribution function or Maxwellian:

$$
\tilde{f}_{\boldsymbol{v}}^{(0)}\left(\boldsymbol{r}, t ; t_{0}\right)=n\left(\boldsymbol{r}, t_{0}\right)\left[\frac{m}{2 \pi T\left(\boldsymbol{r}, t_{0}\right)}\right]^{\frac{3}{2}} \exp \left[-\frac{m\left|\boldsymbol{v}-\boldsymbol{u}\left(\boldsymbol{r}, t_{0}\right)\right|^{2}}{2 T\left(\boldsymbol{r}, t_{0}\right)}\right] \equiv f_{\boldsymbol{v}}^{\mathrm{eq}}\left(\boldsymbol{r} ; t_{0}\right) .
$$

Here, the local density $n\left(\boldsymbol{r}, t_{0}\right)$, local temperature $T\left(\boldsymbol{r}, t_{0}\right)$, and local flux $\boldsymbol{u}\left(\boldsymbol{r}, t_{0}\right)$ are all dependent on the initial time $t_{0}$ and the space coordinate $\boldsymbol{r}$ but independent of time $t$. The 0th-order invariant manifold $\boldsymbol{f}^{(0)}$ is given by the initial condition: $f_{\boldsymbol{v}}^{(0)}\left(\boldsymbol{r}, t_{0}\right)=\tilde{f}_{\boldsymbol{v}}^{(0)}\left(\boldsymbol{r}, t_{0} ; t_{0}\right)=f_{\boldsymbol{v}}^{\text {eq }}\left(\boldsymbol{r} ; t_{0}\right)$. The 0thorder result is summarized as follows:

$$
\left\{\begin{array}{l}
\tilde{\boldsymbol{f}}^{(0)}(t)=\boldsymbol{f}^{\mathrm{eq}} \\
\boldsymbol{f}^{(0)}\left(t_{0}\right)=\boldsymbol{f}^{\mathrm{eq}}
\end{array}\right.
$$

The 1st-order equation now reads

$$
\frac{\partial}{\partial t} \tilde{\boldsymbol{f}}^{(1)}=A \tilde{\boldsymbol{f}}^{(1)}+\boldsymbol{F}
$$


where the time evolution operator $A$ and the inhomogeneous term $\boldsymbol{F}$ are defined by

$$
\left.A_{\boldsymbol{v} \boldsymbol{k}} \equiv \frac{\partial}{\partial f_{\boldsymbol{k}}} I[f] \boldsymbol{v}\right|_{\boldsymbol{f}=\boldsymbol{f}^{\mathrm{eq}}}, \quad F \boldsymbol{v} \equiv-\boldsymbol{v} \cdot \boldsymbol{\nabla} f_{\boldsymbol{v}}^{\mathrm{eq}}
$$

respectively.

As mentioned above, we must clarify the properties of the linear operator $A$ to proceed further. For this purpose, we convert $A$ to the following operator,

$$
L_{\boldsymbol{v} \boldsymbol{k}} \equiv f_{\boldsymbol{v}}^{\mathrm{eq}-1} A_{\boldsymbol{v} \boldsymbol{k}} f_{\boldsymbol{k}}^{\mathrm{eq}}=-\sum_{\boldsymbol{v}_{\mathbf{1}}} \sum_{\boldsymbol{v}_{\mathbf{2}}} \sum_{\boldsymbol{v}_{\mathbf{3}}} \omega\left(\boldsymbol{v}, \boldsymbol{v}_{\mathbf{1}} \mid \boldsymbol{v}_{\mathbf{2}}, \boldsymbol{v}_{\mathbf{3}}\right) f_{\boldsymbol{v}_{\mathbf{1}}}^{\mathrm{eq}}\left(\delta_{\boldsymbol{v} \boldsymbol{k}}+\delta_{\boldsymbol{v}_{1} \boldsymbol{k}}-\delta_{\boldsymbol{v}_{\mathbf{2}} \boldsymbol{k}}-\delta_{\boldsymbol{v}_{\mathbf{3}} \boldsymbol{k}}\right),
$$

which is called the collision operator [17, 19].

Let us define the inner product between arbitrary vectors, $\boldsymbol{\varphi}$ and $\boldsymbol{\psi}$ by

$$
\langle\varphi, \psi\rangle \equiv \sum_{\boldsymbol{v}} f_{\boldsymbol{v}}^{\mathrm{eq}} \varphi \boldsymbol{v} \psi \boldsymbol{v}
$$

We remark that this definition of the inner product is more adequate than that given in [12. A nice point is that $L$ becomes self-adjoint with this inner product; $\langle\boldsymbol{\varphi}, L \boldsymbol{\psi}\rangle=\langle L \boldsymbol{\varphi}, \boldsymbol{\psi}\rangle$. It is essential for the following discussions that $L$ has the zero modes and $\operatorname{dim}[\operatorname{Ker} L]=5[20]$ :

$$
L \boldsymbol{\varphi}_{\alpha}^{0}=\mathbf{0}, \quad(\alpha=0,1,2,3,4)
$$

where the normalized five vectors $\boldsymbol{\varphi}_{\alpha}^{0}$ are given as follows with $\boldsymbol{\delta} \boldsymbol{v} \equiv \boldsymbol{v}-\boldsymbol{u}$;

$$
\begin{aligned}
\varphi_{0}^{0} \boldsymbol{v} & \equiv \frac{1}{\sqrt{n}}, \\
\varphi_{i}^{0} \boldsymbol{v} & \equiv \frac{1}{\sqrt{n}} \sqrt{\frac{m}{T}} \delta v^{i} \text { for } i=1,2,3, \\
\varphi_{4}^{0} \boldsymbol{v} & \equiv \frac{1}{\sqrt{n}} \sqrt{\frac{2}{3}}\left(\frac{m}{2 T}|\boldsymbol{\delta} \boldsymbol{v}|^{2}-\frac{3}{2}\right), .
\end{aligned}
$$

with $\left\langle\varphi_{\alpha}^{0}, \varphi_{\beta}^{0}\right\rangle=\delta_{\alpha \beta}$. The other eigenvalues are found to be negative; in fact one can show that $\langle\boldsymbol{\varphi}, L \varphi\rangle \leq 0$ for all $\boldsymbol{\varphi}$, which means that the kinetic dynamics near the 0th-order solution $\boldsymbol{f}^{\text {eq }}$ has an attractive slow manifold.

Next we define the following projection operator $P$ onto the kernel of $L$,

$$
[P \psi]_{\boldsymbol{v}} \equiv \sum_{\alpha=0}^{4} \varphi_{\alpha}^{0} \boldsymbol{v}\left\langle\boldsymbol{\varphi}_{\alpha}^{0}, \boldsymbol{\psi}\right\rangle
$$

and introduce $Q \equiv 1-P$ as the projection operator to the space complement to $\operatorname{Ker} L$.

Multiplying Eq.(5.14) by the inverse matrix of $f^{\text {eq }}$, we have

$$
\frac{\partial}{\partial t}\left(f^{\mathrm{eq}-1} \tilde{\boldsymbol{f}}^{(1)}\right)=L\left(f^{\mathrm{eq}-1} \tilde{\boldsymbol{f}}^{(1)}\right)+\left(f^{\mathrm{eq}-1} \boldsymbol{F}\right),
$$

where $f_{\boldsymbol{v} \boldsymbol{k}}^{\mathrm{eq}} \equiv f_{\boldsymbol{v}}^{\mathrm{eq}} \delta_{\boldsymbol{v} \boldsymbol{k}}$. The 1 st-order solution is readily obtained as

$$
\tilde{\boldsymbol{f}}^{(1)}(t)=e^{\left(t-t_{0}\right) A}\left[\boldsymbol{f}^{(1)}\left(t_{0}\right)+A^{-1} \bar{Q} \boldsymbol{F}\right]+\left(t-t_{0}\right) \bar{P} \boldsymbol{F}-A^{-1} \bar{Q} \boldsymbol{F},
$$

where $\bar{P} \equiv f^{\text {eq }} P f^{\text {eq-1 }}$ and $\bar{Q} \equiv f^{\text {eq }} Q f^{\text {eq-1 }}$. 
The first order initial value is now determined so that the would-be fast mode disappear, which in turn gives the deformation of the invariant manifold and thereby the 1st-order invariant manifold. Thus we have for the the 1st-order solution

$$
\left\{\begin{array}{l}
\boldsymbol{f}^{(1)}\left(t_{0}\right)=-A^{-1} \bar{Q} \boldsymbol{F}, \\
\tilde{\boldsymbol{f}}^{(1)}(t)=\left(t-t_{0}\right) \bar{P} \boldsymbol{F}-A^{-1} \bar{Q} \boldsymbol{F} .
\end{array}\right.
$$

Then the 2nd-order equation reads

$$
\frac{\partial}{\partial t} \tilde{\boldsymbol{f}}^{(2)}=A \tilde{\boldsymbol{f}}^{(2)}+\left(t-t_{0}\right) \boldsymbol{H}+\boldsymbol{I}
$$

where

$$
H_{\boldsymbol{v}} \equiv-\boldsymbol{v} \cdot \boldsymbol{\nabla}[\bar{P} \boldsymbol{F}]_{\boldsymbol{v}}, \quad I_{\boldsymbol{v}} \equiv \boldsymbol{v} \cdot \boldsymbol{\nabla}\left[A^{-1} \bar{Q} \boldsymbol{F}\right]_{\boldsymbol{v}}
$$

The solution to this equation is found to be

$$
\begin{aligned}
\tilde{\boldsymbol{f}}^{(2)}(t)= & e^{\left(t-t_{0}\right) A}\left[\boldsymbol{f}^{(2)}\left(t_{0}\right)+A^{-2} \bar{Q} \boldsymbol{H}+A^{-1} \bar{Q} \boldsymbol{I}\right] \\
& +\frac{1}{2}\left(t-t_{0}\right)^{2}[\bar{P} \boldsymbol{H}]+\left(t-t_{0}\right)\left[\bar{P} \boldsymbol{I}-A^{-1} \bar{Q} \boldsymbol{H}\right]-\left[A^{-2} \bar{Q} \boldsymbol{H}+A^{-1} \bar{Q} \boldsymbol{I}\right] .
\end{aligned}
$$

Then the 2nd-order results are summarized as follows:

$$
\left\{\begin{array}{l}
\boldsymbol{f}^{(2)}\left(t_{0}\right)=-\left[A^{-2} \bar{Q} \boldsymbol{H}+A^{-1} \bar{Q} \boldsymbol{I}\right] \\
\tilde{\boldsymbol{f}}^{(2)}(t)=\frac{1}{2}\left(t-t_{0}\right)^{2}[\bar{P} \boldsymbol{H}]+\left(t-t_{0}\right)\left[\bar{P} \boldsymbol{I}-A^{-1} \bar{Q} \boldsymbol{H}\right]-\left[A^{-2} \bar{Q} \boldsymbol{H}+A^{-1} \bar{Q} \boldsymbol{I}\right] .
\end{array}\right.
$$

As a result of the above order-by-order analysis, the invariant manifold and the approximate solution up to the 2 nd order are found to be

$$
\begin{aligned}
\boldsymbol{f}\left(t_{0}\right)= & \boldsymbol{f}^{\mathrm{eq}}-\epsilon A^{-1} \bar{Q} \boldsymbol{F}-\epsilon^{2}\left[A^{-2} \bar{Q} \boldsymbol{H}+A^{-1} \bar{Q} \boldsymbol{I}\right] \\
\tilde{\boldsymbol{f}}(t)= & \boldsymbol{f}^{\mathrm{eq}}+\epsilon\left(\left(t-t_{0}\right) \bar{P} \boldsymbol{F}-A^{-1} \bar{Q} \boldsymbol{F}\right) \\
& +\epsilon^{2}\left(\frac{1}{2}\left(t-t_{0}\right)^{2}[\bar{P} \boldsymbol{H}]+\left(t-t_{0}\right)\left[\bar{P} \boldsymbol{I}-A^{-1} \bar{Q} \boldsymbol{H}\right]-\left[A^{-2} \bar{Q} \boldsymbol{H}+A^{-1} \bar{Q} \boldsymbol{I}\right]\right)
\end{aligned}
$$

Notice the appearance of secular terms in (5.31).

\subsection{Procedure 3 : envelope equation or RG equation}

Eq. (5.31) shows that the local approximate solution moves away from the invariant manifold as $\left|t-t_{0}\right|$ becomes large owing to the secular terms. The appearance of the secular terms invalidates the perturbation expansion of the solution around $t \simeq t_{0}$. This evolution is described by the microscopic time described by the kinetic equation Eq.(5.5). We can obtain the global solution valid in a global domain by constructing the envelope of these diverging local solutions parametrized by $t_{0}$. The envelope equation or the $R G$ equation reads:

$$
\left.\frac{\partial}{\partial t_{0}} \tilde{\boldsymbol{f}}(t)\right|_{t_{0}=t}=\mathbf{0} \quad \text { or }\left.\quad \frac{\partial}{\partial t_{0}} \tilde{f} \boldsymbol{v}\left(\boldsymbol{r}, t ; t_{0}\right)\right|_{t_{0}=t}=0
$$


which is reduced to

$$
0=\dot{f}_{\boldsymbol{v}}^{\mathrm{eq}}-\epsilon[\bar{P} \boldsymbol{F}]_{\boldsymbol{v}}-\epsilon^{2}\left[\bar{P} \boldsymbol{I}-A^{-1} \bar{Q} \boldsymbol{H}\right]_{\boldsymbol{v}}-\frac{\partial}{\partial t}\left\{\epsilon\left[A^{-1} \bar{Q} \boldsymbol{F}\right]_{\boldsymbol{v}}+\epsilon^{2}\left[A^{-2} \bar{Q} \boldsymbol{H}+A^{-1} \bar{Q} \boldsymbol{I}\right]_{\boldsymbol{v}}\right\} .
$$

This RG equation is an equation of motion governing the time evolution of the five slow variables $n(\boldsymbol{r}, t), T(\boldsymbol{r}, t)$ and $\boldsymbol{u}(\boldsymbol{r}, t)$ in $\boldsymbol{f}^{\mathrm{eq}}$. If this equation is solved exactly and these variables are substituted in (5.30) at $t_{0}=t$, we can construct the macroscopic time evolution of the oneparticle distribution function $\boldsymbol{f}(t)$. In the discussion below we reduce the master equation (5.33) to a five-dimensional coupled equation. Applying the projection operator $\bar{P}$ from the left of (15.33) we obtain the following:

$$
0=\left[\bar{P} \dot{\boldsymbol{f}}^{\mathrm{eq}}\right]_{\boldsymbol{v}}-\epsilon[\bar{P} \boldsymbol{F}]_{\boldsymbol{v}}-\epsilon^{2}[\bar{P} \boldsymbol{I}]_{\boldsymbol{v}}-\left[\bar{P} \frac{\partial}{\partial t}\left(\epsilon A^{-1} \bar{Q} \boldsymbol{F}+\epsilon^{2}\left(A^{-2} \bar{Q} \boldsymbol{H}+A^{-1} \bar{Q} \boldsymbol{I}\right)\right)\right]_{\boldsymbol{v}}
$$

We notice that the time-derivative does hit to the linear operator $A$ which depends on the zero-th order distribution function $\boldsymbol{f}^{\mathrm{eq}}$, which case is in contrast to that treated in the previous section; we remark that this point was not fully recognized in [12].

Now multipling Eq.(5.34) by the zero modes $\varphi_{\alpha}^{0} \boldsymbol{v}$ and summing up it in terms of $\boldsymbol{v}$, we have

$$
0=\sum_{\boldsymbol{v}} \varphi_{\alpha}^{0} \boldsymbol{v} \dot{f}_{\boldsymbol{v}}^{\mathrm{eq}}-\epsilon \sum_{\boldsymbol{v}} \varphi_{\alpha}^{0} \boldsymbol{v} F \boldsymbol{v}-\epsilon^{2} \sum_{\boldsymbol{v}} \varphi_{\alpha}^{0} \boldsymbol{v} I_{\boldsymbol{v}} \text { for } \alpha=0,1,2,3,4
$$

Here we have used the following relations obtained from the definitions (5.17) and (5.22),

$$
\sum_{\boldsymbol{v}} \varphi_{\alpha}^{0} \boldsymbol{v}[\bar{P} \psi]_{\boldsymbol{v}}=\sum_{\boldsymbol{v}} \varphi_{\alpha}^{0} \boldsymbol{v} \psi \boldsymbol{v}, \quad \sum_{\boldsymbol{v}} \varphi_{\alpha}^{0} \boldsymbol{v}[\bar{Q} \psi]_{\boldsymbol{v}}=0
$$

and the equality

$$
\sum_{\boldsymbol{v}} \varphi_{\alpha}^{0} \boldsymbol{v} \frac{\partial}{\partial t}\left\{\epsilon\left[A^{-1} \bar{Q} \boldsymbol{F}\right]_{\boldsymbol{v}}+\epsilon^{2}\left[A^{-2} \bar{Q} \boldsymbol{H}+A^{-1} \bar{Q} \boldsymbol{I}\right]_{\boldsymbol{v}}\right\}=0
$$

which follows from the fact that $\dot{\varphi}_{\alpha}^{0} \in \operatorname{Ker} L$. Notice that (5.35) has the same information as the RG equation (5.33).

\subsection{Explicit reduction to Navier-Stokes equation; transport coeffi- cients}

We now show that Eq. (5.35) is nothing but the Navier-Stokes equation[20. Performing the summation in terms of $\boldsymbol{v}$, we find that the first and second terms of Eq.(5.35) are evaluated to be

$$
\begin{aligned}
\sum_{\boldsymbol{v}} \varphi_{0}^{0} \boldsymbol{v} \dot{f}_{\boldsymbol{v}}^{\mathrm{eq}} & =\left(\frac{1}{\sqrt{n}} \frac{1}{m}\right) m \dot{n}, \\
\sum_{\boldsymbol{v}} \varphi_{i}^{0} \boldsymbol{v} \dot{f}_{\boldsymbol{v}}^{\mathrm{eq}} & =\left(\frac{1}{\sqrt{n}} \sqrt{\frac{m}{T}} \frac{1}{m}\right) m n \dot{u}^{i} \text { for } i=1,2,3, \\
\sum_{\boldsymbol{v}} \varphi_{4}^{0} \boldsymbol{v} \dot{f}_{\boldsymbol{v}}^{\mathrm{eq}} & =\left(\frac{1}{\sqrt{n}} \sqrt{\frac{2}{3}} \frac{1}{T}\right) n \frac{3}{2} \dot{T}
\end{aligned}
$$

and

$$
\begin{aligned}
\sum_{\boldsymbol{v}} \varphi_{0}^{0} \boldsymbol{v} F_{\boldsymbol{v}} & =-\left(\frac{1}{\sqrt{n}} \frac{1}{m}\right) m \boldsymbol{\nabla} \cdot(n \boldsymbol{u}), \\
\sum_{\boldsymbol{v}} \varphi_{i}^{0} \boldsymbol{v} F_{\boldsymbol{v}} & =-\left(\frac{1}{\sqrt{n}} \sqrt{\frac{m}{T}} \frac{1}{m}\right)\left(m n \boldsymbol{u} \cdot \nabla u^{i}+\nabla^{i}(n T)\right) \text { for } i=1,2,3, \\
\sum_{\boldsymbol{v}} \varphi_{4}^{0} \boldsymbol{v} F_{\boldsymbol{v}} & =-\left(\frac{1}{\sqrt{n}} \sqrt{\frac{2}{3}} \frac{1}{T}\right)\left(n \boldsymbol{u} \cdot \boldsymbol{\nabla}\left(\frac{3}{2} T\right)+n T \boldsymbol{\nabla} \cdot \boldsymbol{u}\right)
\end{aligned}
$$


In a similar way, the third terms of Eq. (5.35) are evaluated to be

$$
\begin{aligned}
\sum_{\boldsymbol{v}} \varphi_{0}^{0} \boldsymbol{v} I_{\boldsymbol{v}} & =0 \\
\sum_{\boldsymbol{v}} \varphi_{i}^{0} \boldsymbol{v}^{I} \boldsymbol{v} & =\left(\frac{1}{\sqrt{n}} \sqrt{\frac{m}{T}} \frac{1}{m}\right) \nabla^{j}\left\langle\boldsymbol{T}^{i j}, L^{-1} Q f^{\mathrm{eq}-1} \boldsymbol{F}\right\rangle \text { for } i=1,2,3 \\
\sum_{\boldsymbol{v}} \varphi_{4}^{0} \boldsymbol{v}^{I} \boldsymbol{v} & =\left(\frac{1}{\sqrt{n}} \sqrt{\frac{2}{3}} \frac{1}{T}\right)\left(\nabla^{i}\left\langle\boldsymbol{J}^{i}, L^{-1} Q f^{\mathrm{eq}-1} \boldsymbol{F}\right\rangle+\left\langle\boldsymbol{T}^{i j}, L^{-1} Q f^{\mathrm{eq}-1} \boldsymbol{F}\right\rangle \frac{1}{2}\left(\nabla^{i} u^{j}+\nabla^{j} u^{i}\right)\right),
\end{aligned}
$$

where $\boldsymbol{T}^{i j}$ and $\boldsymbol{J}^{i}$ are defined as

$$
T_{\boldsymbol{v}}^{i j} \equiv m \delta v^{i} \delta v^{j}, \quad J_{\boldsymbol{v}}^{i} \equiv\left(\frac{1}{2} m|\boldsymbol{\delta} \boldsymbol{v}|^{2}-\frac{5}{2} T\right) \delta v^{i} .
$$

Here we have replaced $\varphi_{4}^{0} \boldsymbol{v} \delta v^{i}$ by $J_{\boldsymbol{v}}^{i}$ because $\delta v^{i}$ belongs to Ker $L$ and its inner product with $\left[L^{-1} Q f^{\text {eq-1 }} \boldsymbol{F}\right]_{\boldsymbol{v}}$ is null.

To proceed further, the explicit representation of $\left[L^{-1} Q f^{\text {eq-1 }} \boldsymbol{F}\right]_{\boldsymbol{v}}$ is necessary. By using the projection operator (5.22), we have

$$
\left[L^{-1} Q f^{\mathrm{eq}-1} \boldsymbol{F}\right]_{\boldsymbol{v}}=-\frac{1}{T} \sum_{\boldsymbol{k}} L_{\boldsymbol{v} \boldsymbol{k}}^{-1}\left[T_{\boldsymbol{k}}^{i j} \frac{1}{2}\left(\nabla^{i} u^{j}+\nabla^{j} u^{i}-\frac{2}{3} \delta^{i j} \boldsymbol{\nabla} \cdot \boldsymbol{u}\right)+J_{\boldsymbol{k}}^{i} \nabla^{i} \ln T\right] .
$$

Following the above equation and the space rotational symmetry [17, 19], we arrive at

$$
\begin{aligned}
& \left\langle\boldsymbol{T}^{i j}, L^{-1} Q f^{\mathrm{eq}-1} \boldsymbol{F}\right\rangle=2 \eta \frac{1}{2}\left(\nabla^{i} u^{j}+\nabla^{j} u^{i}-\frac{2}{3} \delta^{i j} \nabla \cdot \boldsymbol{u}\right), \\
& \left\langle\boldsymbol{J}^{i}, L^{-1} Q f^{\mathrm{eq}-1} \boldsymbol{F}\right\rangle=\lambda \nabla^{i} T,
\end{aligned}
$$

where $\eta$ and $\lambda$ are the so-called transport coefficients defined by

$$
\begin{aligned}
& \eta \equiv-\frac{1}{T} \sum_{\boldsymbol{v} \boldsymbol{k}} f_{\boldsymbol{v}}^{\mathrm{eq}} T_{\boldsymbol{v}}^{12} L_{\boldsymbol{v} \boldsymbol{k}}^{-1} T_{\boldsymbol{k}}^{12}=\frac{1}{T} \int_{0}^{\infty} d \tau\left\langle\boldsymbol{T}^{12}(0), \boldsymbol{T}^{12}(\tau)\right\rangle, \quad\left[\boldsymbol{T}^{i j}(\tau)\right]_{\boldsymbol{v}} \equiv \sum_{\boldsymbol{k}}\left[e^{L \tau}\right]_{\boldsymbol{v} \boldsymbol{k}} T_{\boldsymbol{k}}^{i j} \\
& \lambda \equiv-\frac{1}{T^{2}} \sum_{\boldsymbol{v} \boldsymbol{k}} f_{\boldsymbol{v}}^{\mathrm{eq}} J_{\boldsymbol{v}}^{1} L_{\boldsymbol{v} \boldsymbol{k}}^{-1} J_{\boldsymbol{k}}^{1}=\frac{1}{T^{2}} \int_{0}^{\infty} d \tau\left\langle\boldsymbol{J}^{1}(0), \boldsymbol{J}^{1}(\tau)\right\rangle, \quad\left[\boldsymbol{J}^{i}(\tau)\right]_{\boldsymbol{v}} \equiv \sum_{\boldsymbol{k}}\left[e^{L \tau}\right]_{\boldsymbol{v} \boldsymbol{k}} J_{\boldsymbol{k}}^{i}
\end{aligned}
$$

We note that the transport coefficients, shear viscosity $\eta$ and heat conductivity $\lambda$, are obtained as the correlation function of the microscopic currents (5.47) like Kubo formula [18].

Putting back $\epsilon=1$, the reduced RG equations (5.35) are found to be

$$
\begin{aligned}
\dot{n}+\boldsymbol{\nabla} \cdot(n \boldsymbol{u}) & =0, \\
m n \dot{u}^{i}+m n \boldsymbol{u} \cdot \boldsymbol{\nabla} u^{i} & =-\nabla^{j} P^{j i} \text { for } i=1,2,3, \\
n \dot{e}+n \boldsymbol{u} \cdot \boldsymbol{\nabla} \epsilon & =-\boldsymbol{\nabla} \cdot \boldsymbol{J}-P^{i j} D^{i j},
\end{aligned}
$$

where

$$
\begin{aligned}
& e \equiv \frac{3}{2} T, \quad p \equiv n T, \quad D^{i j} \equiv \frac{1}{2}\left(\nabla^{i} u^{j}+\nabla^{j} u^{i}\right), \\
& P^{i j} \equiv \delta^{i j} p-2 \eta D^{i j}-\left(-\frac{2}{3} \eta\right) \delta^{i j} \nabla \cdot \boldsymbol{u}, \quad \boldsymbol{J} \equiv-\lambda \nabla T .
\end{aligned}
$$

These equations (5.53)-(5.55) is identified with the fluid dynamic equations with dissipation, i.e., the Navier-Stokes equation 20].

In summary, we have shown that the Navier-Stokes equations can be neatly reproduced as the fluid dynamical limit of the Boltzmann equation in the RG method. 


\section{Summary and concluding remarks}

We have shown that the RG gives a powerful and systematic method for the reduction of the dynamics and also provides a transparent way for the construction of the attractive slow manifold. We have indicated the relation of the underlying mathematics of the RG method with the classical theory of envelopes in mathematical analysis. Although the uses of envelopes for physics problem was first noted by M. Suzuki in the theory of CAM(Coherent Anomaly Method) 23 for the critical phenomena in statistical physics, the usefulness of the notion of envelopes was not well recognized.

We have obtained the Navier-Stokes equation from the Boltzmann equation by applying the RG method: We have worked out for constructing the projection operators and thus explicitly given the forms of the transport coefficients in terms of the one-particle distribution function.

The RG method presented here has a wide range of applicabilities even being confined to the transport equations [12]: It can be applied to obtain the Boltzmann equation from the Liouville equation. The Focker-Planck equation is equally obtained from the Langevin equation by this

method. Furthermore, the further reduction of the Focker-Planck equation can be also done by the RG method.

As for the reduction of the hydrodynamic equation from the Boltzmann equation, it would be interesting to apply the present method to the relativistic case. We also mention that the present method is also applicable to extract the critical slow dynamics around the critical point of phase transitions [1]. 11. It would be also interesting to apply the method for extracting the slow dynamics, say, around the QCD critical end point [24].

\section{Acknowledgments}

T. K. is supported by Grant-in-Aide for Scientific Research by Monbu-Kagaku-sho (No. 17540250). This work is supported in part by a Grant-in-Aid for the 21st Century COE "Center for Diversity and Universality in Physics".

\section{References}

[1] E.C.G. Stuckelberg and A. Petermann, Helv. Phys. Acta 26(1953) 499; M. Gell-Mann and F. E. Low, Phys. Rev. 95 (1953) 1300.

As a review article, J. Zinn-Justin, Quantum Field Theory and Critical Phenomena (Clarendon Press, Oxford, 1989).

[2] K.G. Wilson, Phys. Rev. D3 (1971), 1818

[3] F. Wegner and A. Houghton, Phys. Rev. A8 (1973), 401

[4] S. Weinberg, "The Quantum Theory of Field II", Cambrige U.P., 1996

[5] C.M. Bender and S.A. Orszag, Advanced Mathematical Methods for Scientists and Engineers (McGraw-Hill, 1978).

[6] L.Y. Chen, N. Goldenfeldand Y. Oono, Phys. Rev. Lett.73(1994)1311; Phys. Rev. E 54 (1996) 376; See also J. Bricmont and A. Kupianien, Commun. Math. Phys. 150 (1992) 193.

[7] T.Kunihiro, Prog.Theor.Phys. 94, 503(1995); 95, 835(1996)(E). 
[8] T.Kunihiro, Jpn. J. Ind. Appl. Math. 14, 51 (1997).

[9] T.Kunihiro, Prog. Theor. Phys. 97, 179 (1997).

[10] T. Kunihiro and J. Matsukidaira, Phys. Rev. E57 (1998), 4817.

[11] S.-I. Ei, K. Fujii and T. Kunihiro, Ann. Phys. 280 (2000), 236.

[12] Y. Hatta and T. Kunihiro, Ann. Phys. 298 (2002) 24

[13] T. Hatsuda, T. Kunihiro and T. Tanaka, Phys. Rev. Lett.78 (1997) 3229.

[14] Y. Kuramoto, Prog. Theor. Phys. Suppl. 99 (1989) 244; Bussei Kenkyu 49(1987) 299.

[15] N. N. Bogoliubov and Y. A. Mitropolsky, Asymptotic Methods in the Theory of Nonlinear Oscillations, Gordon and Breach, 1961.

[16] N.N. Bogoliubov, in 'Studies in Statistical Mechanics' (J. de Boer and G.E. Uhlenbeck, Eds.), Vol.2, (North-Holland, Amsterdam, 1962).

[17] S. Chapman and T.G. Cowling, "The Mathematical Theory of Non-Uniform Gases" (3rd ed.) Cambridge U.P., 1970.

[18] R. Kubo, M. Toda and N. Hashitsume, Statistical Physics II Non-equilibrium Statistical Mechanics (Springer-Verlag, 1985).

[19] K. Kawasaki, Non-equilibrium and Phase Transition - Statistical Physics in Meso Scales, chap. 7 (Asakura Shoten, 2000), in Japanese.

[20] P. Resibois, J. Stat. Phys. 2,(1970), 21; R. Balescu, Equilibrium and Nonequilibrium Statistical Mechanics, (Wiley, New York, 1975); L.E. Reichl, Modern Course in Statistical Physics, chap. 11, 2nd ed. (John Wiley and Sons, 1998).

[21] L. Boltzmann, "Lectures on Gas Theory," University of California Press, Berkeley, 1964.

[22] E. M. Lifshitz and L. P. Pitaevskii, "Physical Kinetics," Butterworth-Heinemann, 1981.

[23] M. Suzuki, J. Phys. Soc. Jpn., 55 (1986), 4205; Mean Field, Fluctuations and Systematics, M. Suzuki et al ed. (World Scientific, 1995)

[24] M. A. Stephanov, K. Rajagopal and E. V. Shuryak, Phys. Rev. Lett. 81(1998), 4816; D. T. Son and M. A. Stephanov, Phys. Rev. D70 (2004), 056001; see also, K. Ohnishi and T. Kunihiro, nucl-th/0509044. 